\title{
Mapping of language and motor function during awake neurosurgery with intraoperative optical imaging
}

\author{
Martin Oelschlägel, Dipl-Ing, ${ }^{1}$ Tobias Meyer, Dr rer medic, ${ }^{2}$ Ute Morgenstern, Dr-Ing, ${ }^{3}$ \\ Hannes Wahl, Dipl-Ing, ${ }^{4}$ Johannes Gerber, Dr med, ${ }^{4}$ Gilfe Reiß, Dr med, ${ }^{6}$ \\ Edmund Koch, Dr rer nat, ${ }^{1}$ Gerald Steiner, Dr-Ing et rer nat, ${ }^{1}$ Matthias Kirsch, Dr med, ${ }^{5}$ \\ Gabriele Schackert, Dr med, ${ }^{6}$ and Stephan B. Sobottka, Dr med ${ }^{6}$
}

${ }^{1}$ Clinical Sensoring and Monitoring, Department of Anesthesiology and Intensive Care Medicine, Faculty of Medicine Carl Gustav Carus, Technische Universität Dresden; ${ }^{2} \mathrm{ABX}$-CRO Advanced Pharmaceutical Services Forschungsgesellschaft mbH, Dresden; ${ }^{3}$ Institute of Biomedical Engineering, Faculty of Electrical and Computer Engineering, Technische Universität Dresden; ${ }^{4}$ nstitute and Polyclinic of Diagnostic and Interventional Neuroradiology, Carl Gustav Carus University Hospital, Technische Universität Dresden; ${ }^{5}$ Department of Neurosurgery, Asklepios Kliniken Schildautal Seesen; and ${ }^{6}$ Department of Neurosurgery, Carl Gustav Carus University Hospital, Technische Universität Dresden, Saxony, Germany

\begin{abstract}
Intraoperative optical imaging $(\mathrm{IOI})$ is a marker-free, contactless, and noninvasive imaging technique that is able to visualize metabolic changes of the brain surface following neuronal activation. Although it has been used in the past mainly for the identification of functional brain areas under general anesthesia, the authors investigated the potential of the method during awake surgery. Measurements were performed in 10 patients who underwent resection of lesions within or adjacent to cortical language or motor sites. IOI was applied in 3 different scenarios: identification of motor areas by using finger-tapping tasks, identification of language areas by using speech tasks (overt and silent speech), and a novel approach - the application of $\mathrm{IOI}$ as a feedback tool during direct electrical stimulation (DES) mapping of language. The functional maps, which were calculated from the IOI data (activity maps), were qualitatively compared with the functional MRI (fMRI) and the electrophysiological testing results during the surgical procedure to assess their potential benefit for surgical decision-making.

The results reveal that the intraoperative identification of motor sites with $\mathrm{IOI}$ in good agreement with the preoperatively acquired fMRI and the intraoperative electrophysiological measurements is possible. Because IOI provides spatially highly resolved maps with minimal additional hardware effort, the application of the technique for motor site identification seems to be beneficial in awake procedures. The identification of language processing sites with IOI was also possible, but in the majority of cases significant differences between $\mathrm{FMRI}$, IOI, and DES were visible, and therefore according to the authors' findings the IOI results are too unspecific to be useful for intraoperative decision-making with respect to exact language localization. For this purpose, DES mapping will remain the method of choice.

Nevertheless, the IOI technique can provide additional value during the language mapping procedure with DES. Using a simple difference imaging approach, the authors were able to visualize and calculate the spatial extent of activation for each stimulation. This might enable surgeons in the future to optimize the mapping process. Additionally, differences between tumor and nontumor stimulation sites were observed with respect to the spatial extent of the changes in cortical optical properties. These findings provide further evidence that the method allows the assessment of the functional state of neurovascular coupling and is therefore suited for the delineation of pathologically altered tissue.
\end{abstract}

https://thejns.org/doi/abs/10.3171/2019.11.FOCUS19759

KEYWORDS intraoperative optical imaging; awake brain surgery; direct electrical stimulation; functional magnetic resonance imaging; language mapping; motor function mapping

ABBREVIATIONS $\triangle \mathrm{CBV}=$ changes in cerebral blood volume; $\mathrm{DES}=$ direct electrical stimulation; $\mathrm{FA}=$ flip angle; $\mathrm{fMRI}=$ functional $\mathrm{MRI}$; IOI = intraoperative optical imaging; $\mathrm{RDI}=$ relative difference image.

SUBMITTED September 20, 2019. ACCEPTED November 15, 2019

INCLUDE WHEN CITING DOI: 10.3171/2019.11.FOCUS19759. 
$\mathrm{P}$ REOPERATIVE functional neuroimaging of the human brain is essential to avoid postoperative deficits in patients undergoing surgery adjacent to or within eloquent brain areas. Several different techniques are available, including functional MRI (fMRI), PET, and diffusion-weighted imaging (DWI).,13 Although all of the mentioned methods are invaluable for risk stratification and preoperative planning,,$^{8,9,27}$ one disadvantage of these techniques is that the functional imaging data are acquired preoperatively and matched to the intraoperative site using neuronavigation systems. This approach has the drawback that after craniotomy, due to brain shift, it is unclear how precise the image fusion actually is. Studies have shown that target registration errors up to $5 \mathrm{~mm}$ may occur, depending on the registration method used. ${ }^{14,32}$ Additionally, most of the mentioned methods are still limited in their spatial resolution.

Intraoperative optical imaging (IOI) is a functional imaging technique that is able to overcome those drawbacks. It enables the surgeon to view changes in the cortical optical properties by acquisition and evaluation of camera images from the exposed brain surface. The camera images can be acquired with high temporal and spatial resolution. The noninvasive and contactless imaging technique allows the observation of metabolic changes (changes in cerebral blood volume $[\Delta \mathrm{CBV}]$ and oxygenation) and therefore the investigation of cortical organization ${ }^{12,26,34}$ and neuronal connectivity. 2,35,36 In human subjects, IOI has been extensively used for the exploration, identification, and mapping of somatosensory ${ }^{16,23-25,29}$ areas as well as for the visual cortex. ${ }^{30}$ Furthermore, the method has been used for the delineation of epileptic foci ${ }^{11,31}$ and brain tumors. ${ }^{10,17}$ A more detailed description of the imaging technique and its applications can be found in the study by Prakash et al. ${ }^{22}$ or in a more recent publication by Morone et al. ${ }^{15}$ Concerning language-related observations with IOI in awake patients, especially in comparison to fMRI and direct electrical stimulation (DES) mapping, only a few studies are currently available, ,5,6,20 and the use of IOI for decision-making during tumor resection is still not conclusively clarified.

Therefore, in this study we qualitatively compared the colocalization of speech and motor areas between IOI, fMRI, and DES. Measurements in 10 patients who underwent brain mapping under local anesthesia were performed. Additionally, we present a novel approach: the use of IOI as a tool for visual feedback generation during DES language mapping.

\section{Methods}

\section{Preoperative Imaging (MRI and fMRI)}

Preoperative fMRI was performed on Siemens Sonata 1.5-T (9/10 patients) and Siemens Verio 3-T (1/10 patients) scanners by using an 8-channel head coil. Two different paradigms were used in a block design: finger tapping $(2 / 10)$ and verb generation $(8 / 10)$.

Finger-tapping results were acquired for patients' left and right hands with 2 scans: 1 without tapping and 1 with tapping. The following scanner parameters were used: acquisition matrix $64 \times 64$, TE $54 \mathrm{msec}$, TR $3520 \mathrm{msec}$, flip angle (FA) $90^{\circ}$, and slice thickness $3 \mathrm{~mm}$, with a slice gap of $3.75 \mathrm{~mm}$ and an in-plane resolution of $3 \times 3 \mathrm{~mm}$, for 30 slices and 2 volumes for each hand.

For the verb generation task $5 \times 10$ scans were acquired during a rest trial and $5 \times 10$ scans while performing the task (silent speech). The following acquisition parameters were used: acquisition matrix $64 \times 64$, TE $50 \mathrm{msec}$, TR $3480 \mathrm{msec}, \mathrm{FA} 90^{\circ}$, and slice thickness $3 \mathrm{~mm}$, with a slice gap of $3.75 \mathrm{~mm}$ and an in-plane resolution of $3 \times 3 \mathrm{~mm}$, for 26 slices and 100 volumes, with 2 total measurements per imaging session. The parameters were the same on the 1.5-T and 3-T scanners.

The fMRI data sets were analyzed with SPM for MATLAB (MathWorks), using a corrected significance level of $\mathrm{p}<0.05$ with a cluster size of $>20$ voxels. A high-resolution, T1-weighted structural image (magnetization-prepared rapid acquisition gradient echo [MPRAGE], acquisition matrix $256 \times 168$, TE $2.86 \mathrm{msec}$, TR $5.98 \mathrm{msec}$, FA $10^{\circ}$, isotropic voxel $1 \mathrm{~mm}^{3}$ ) was acquired after functional imaging.

\section{Intraoperative Imaging Procedure}

Different IOI setups were used for the measurements; Table 1 gives an overview regarding the technical components of each setup. In all cases, a camera device had been attached via a beam splitter to the surgical microscope. Light wavelength filtering was performed (monochrome imaging cameras) within the optical path at $\lambda=568 \pm 5$ $\mathrm{nm}$, aiming at blood volume changes $(\triangle \mathrm{CBV})$. Image data sets were acquired with 4-7 frames per second. Parts of the different hardware setups that were used for the measurements are described in detail elsewhere. ${ }^{28,29}$ Table 2 gives an overview of the IOI system that was used for each patient. IOI was performed in all patients directly after craniotomy. The measurements were performed completely contactless and noninvasively (without, e.g., stabilizing glass plates on the cortical surface). The speech task (overt speech), lasting 9 minutes, was the first that was executed by 8 of the 10 patients. The protocol for this task was designed according to the protocols that were used in the past for the stimulation of different peripheral nerves. It comprised a 9-minute scheme with alternating 30-second rest and 30-second naming trials. In 1 patient this scheme was varied, and naming without vocalization (silent speech) was additionally performed. The objects that were to be named were presented intraoperatively to the patient by using timed slides in a presentation that was running on a commercial laptop computer. While the patients were performing the speech task, the objects being shown changed every 5 seconds within the naming ("stimulation") trials. During the 30-second rest trials, a blank slide was shown.

In 2 of the 10 patients we observed the routine DES speech mapping with IOI. For each stimulation site, a data set with 1 minute of imaging data was acquired. Those data sets comprised 15 seconds of baseline images, $5 \mathrm{sec}$ onds of stimulation images, and 40 seconds of poststimulus images, resulting in a total data set length of $1 \mathrm{~min}-$ ute. Stimulations during speech mapping were performed using a stimulation current $\left(\mathrm{I}_{\text {stim }}\right)$ of $6 \mathrm{~mA}$, a stimulation duration $\left(\mathrm{T}_{\text {stim }}\right)$ of 5 seconds, and a stimulation frequency $\left(f_{\text {stim }}\right)$ of $50 \mathrm{~Hz}$ (pulse width [ $\left.\mathrm{T}_{\text {pulse }}\right]$ of $200 \mu \mathrm{sec}$ ). Elec- 
TABLE 1. IOI hardware

\begin{tabular}{|c|c|c|c|}
\hline Hardware & Setup No. 1 & Setup No. 2 & Setup No. 3 \\
\hline Camera & Hamamatsu C4742-96-12G04 & Zeiss AxioCam MRm & Zeiss Trio 610/620 \\
\hline Filtering & $568 \mathrm{~nm}$ & $568 \mathrm{~nm}$ & None (RGB camera) \\
\hline Exposure time & $800 \mathrm{msec}$ & $50 \mathrm{msec}$ & $1 \mathrm{msec}$ \\
\hline Microscope & Möller-Wedel Hi-R 1000 & Zeiss OPMI Pico/Zeiss OPMI Pentero & Zeiss OPMI Pico/Zeiss OPMI Pentero \\
\hline $\begin{array}{l}\text { Illumination (microscope } \\
\text { integrated) }\end{array}$ & Halogen 150 W & Xenon $180 \mathrm{~W} /$ xenon $300 \mathrm{~W}$ & Xenon $180 \mathrm{~W} /$ xenon $300 \mathrm{~W}$ \\
\hline Resolution & $672 \times 512$ pixel $(2 \times 2$ binning mode $)$ & $692 \times 520$ pixel $(2 \times 2$ binning mode $)$ & $1920 \times 1080$ pixel \\
\hline Digitization & 12 bits & 12 bits & 8 bits per color channel \\
\hline Data transfer via & CCU/IEEE 1394 FireWire & IEEE 1394 FireWire & $\begin{array}{l}\text { Frame-grabber board connected to } \\
\text { CCU (Trio } 600 \mathrm{CCU} \text { ) }\end{array}$ \\
\hline
\end{tabular}

$\mathrm{CCU}=$ camera controller unit.

trocorticography was performed parallel to the optical recordings to detect afterdischarges as well as seizures. Therefore, a grid electrode was positioned subdurally outside of the exposed cortical region.

The motor tasks were designed in the same way as the language tasks. Finger tapping and hand opening and closing were performed in case 1 and case 2 , respectively, in 30-second trials over 9 minutes. To validate the IOI measurements, motor areas were intraoperatively identified using phase-reversal measurements of somatosensory evoked potentials and monopolar cortex stimulation.

\section{Patients}

Eight patients with typical MRI appearance of left frontotemporal low-grade glioma and 2 patients with metastases close to the precentral gyrus ( 5 male, 5 female; median age 40.5 years) were included in this study. Informed consent was obtained from all patients. The study was approved by the ethics committee of the Technische Universität Dresden. Table 2 gives an overview of the patient characteristics and the imaging methods applied in each patient.

\section{Data Analysis, Validation, and Assessment of fMRI, DES Mapping, and IOI}

The data evaluation for patients performing 9-minute tasks (speech or motor tasks) was done by using Fourier analysis and is described in detail in previous publications. ${ }^{18,29}$ The resulting IOI activity maps were coregistered to preoperatively acquired anatomical MRI and fMRI data. The registration was manually performed based on anatomical landmarks and data points from the neuronavigation system. The borders of the skull trepanation as well as the different DES sites were typically saved during the surgical procedure to ease the postoperative image registration and fusion process. Based on the registered data sets and their visualization, the agreement of the localization results for the different modalities (IOI, DES, and fMRI) was qualitatively assessed by 3 independent evaluators. A 4-level grading scheme was used for the evaluation, including the levels "no," "low," "moderate," and "high" agreement. A detailed explanation of each level can be found in the footnote of Table 3.

During the language mapping procedure with DES, a difference imaging technique was used to calculate pseu-

TABLE 2. Patient characteristics, functional imaging/mapping methods, and IOI setup used in each case

\begin{tabular}{|c|c|c|c|c|c|c|c|c|c|}
\hline Case No. & Age & Sex & Tumor Location & Histopathology & IOI & Task & IOI w/ DES & fMRI & IOI Hardware Setup No. \\
\hline \multicolumn{10}{|c|}{ Motor Tasks } \\
\hline 1 & 72 & $\mathrm{~F}$ & Lt frontoparietal & Metastasis (bronchial-carcinoma) & Yes & Finger tapping & No & Yes & 3 \\
\hline 2 & 75 & $\mathrm{~F}$ & Rt precentral region & Metastasis (sarcoma) & Yes & $\begin{array}{l}\text { Hand opening/ } \\
\text { closing }\end{array}$ & No & Yes & 3 \\
\hline \multicolumn{10}{|c|}{ Speech Tasks } \\
\hline 3 & 41 & M & Lt insula & Astrocytoma grade III & Yes & $\begin{array}{l}\text { Silent speech, } \\
\text { overt speech }\end{array}$ & No & Yes & 1 \\
\hline 4 & 34 & M & Lt temporal/insula & Oligoastrocytoma grade II & Yes & Overt speech & No & Yes & 2 \\
\hline 5 & 36 & M & Lt frontotemporal & Astrocytoma grade II & Yes & Overt speech & No & Yes & 2 \\
\hline 6 & 53 & M & Lt temporal & Oligodendroglioma grade III & Yes & Overt speech & No & Yes & 2 \\
\hline 7 & 36 & $\mathrm{~F}$ & Lt frontal & Oligoastrocytoma grade III & Yes & Overt speech & No & Yes & 2 \\
\hline 8 & 38 & $\mathrm{~F}$ & Lt frontal & Oligoastrocytoma grade II & Yes & Overt speech & No & Yes & 2 \\
\hline 9 & 40 & $\mathrm{~F}$ & Lt frontal & Astrocytoma grade III & Yes & Overt speech & Yes & Yes & 2 \\
\hline 10 & 53 & M & Lt frontal & Glioblastoma multiforme grade IV & Yes & Overt speech & Yes & Yes & 2 \\
\hline
\end{tabular}


TABLE 3. Agreement of the different modalities

\begin{tabular}{|c|c|c|c|c|}
\hline Case No., Task & fMRI \& EM & IOI \& fMRI & IOI \& EM & Remarks \\
\hline \multicolumn{5}{|l|}{ Motor tasks } \\
\hline 1, finger tapping & $* * *$ & $* * *$ & $* * *$ & Additional activity on postcentral gyrus in red channel; see Fig. 2 \\
\hline 2 , hand opening/closing & *** & *** & *** & \\
\hline Case No., Task & fMRI \& DES & IOI \& fMRI & IOI \& DES & Remarks \\
\hline \multicolumn{5}{|l|}{ Speech tasks } \\
\hline 3, overt & \multirow{2}{*}{ * } & $* * *$ & - & \multirow{2}{*}{$\begin{array}{l}\text { IOI w/ naming correlates more } w / \text { fMRI, \& IOI w/ verbalization cor- } \\
\text { relates more w/ DES; see Fig. } 3\end{array}$} \\
\hline 3 , silent & & ** & ** & \\
\hline 4 , overt & - & * & * & Functional activity w/in tumor (IOI \& DES) \\
\hline 5 , overt & $* *$ & ** & ** & \\
\hline 6 , overt & ** & - & - & Almost no activity visible w/in IOI \\
\hline 7, overt & ** & ** & ** & \\
\hline 8 , overt & - & - & * & Almost no activity visible w/in fMRI \\
\hline 9, overt & ** & - & ** & \\
\hline 10, overt & $* *$ & ** & $* * *$ & \\
\hline
\end{tabular}

$\mathrm{EM}=$ electrophysiological measurements (i.e., phase reversal of somatosensory evoked potentials and monopolar cortex stimulation); ${ }^{*}=$ low agreement: some areas match, but most do not; ${ }^{* *}=$ moderate agreement: differences between both modalities, but most areas match; ${ }^{* *}=$ high agreement: identification of nearly the same areas by both modalities; $-=$ no agreement: completely different areas identified by both modalities.

docolored activity maps that represent the spatial extent of metabolic changes $(\triangle \mathrm{CBV})$. The relative difference image (RDI) was calculated for each stimulation site by using a mean baseline image $\left(I_{\text {base }}\right)$ and a mean poststimulus image $\left(I_{p s}\right)$ according to the following formula:

$$
R D I=\frac{\overline{I_{p s}}-\overline{I_{\text {base }}}}{\overline{I_{\text {base }}}} \text {. }
$$

As we have described elsewhere, ${ }^{17}$ the highest extent of metabolic changes is visible within the IOI data directly after stimulation ends. Therefore, $I_{\text {base }}$ was calculated from images acquired 10 seconds prior to the stimulation's start, and $I_{p s}$ from images that were recorded 10 seconds directly after the stimulation's end. A more detailed description of this methodology can be found in Oelschlägel et al. ${ }^{17}$ To calculate the extent of activation, we used the image processing chain that is shown in detail in Fig. 1. The area (A) of the final activation (Fig. 1F) was calculated from the image data based on the known interelectrode distance (approximately $0.8 \mathrm{~cm}$ ) of the bipolar electrode.

\section{Results}

\section{Functional Measurements (motor and speech tasks)}

The motor tasks section of Table 3 shows in detail the agreement of IOI and fMRI with respect to motor tasks. Using the IOI method, we were able to induce locally delineated activity on the precentral gyrus (motor cortex) in both patients (one patient performed finger tapping and the other patient did hand opening and closing). Comparing the different observation wavelengths of the RGB camera (different color channels) in the patient in case 1, additional activity on the precentral gyrus (sensory cortex) is visible especially in the red channel (deoxyhemoglobindominated signal). Moreover, the red channel activity map emphasizes large venous structures, whereas the blue and green channel maps tend to be more focused on parenchymal changes of the cortical optical properties (Fig. 2). The results of IOI correspond with the preoperatively acquired fMRI as well as with the intraoperative phase-reversal measurements and electrophysiological mapping.

The speech tasks section of Table 3 shows the agreement for language localization of the different imaging modalities. In 5 of 8 patients, an agreement between the speech localization of IOI and fMRI is observable. The other 3 patients' measurements reveal no correlation between both modalities, caused in 2 cases by weak fMRI or weak IOI activation and in 1 patient by completely different activation sites. Comparing IOI and DES, 7 of 8 patients do show an agreement. In 1 patient, no correspondence between the imaging modalities was visible. Figure 3 shows the detailed results for case 3 with a low-grade astrocytoma within the left insula region. A speech arrest was located at marker 7 (blue). A seizure was induced at marker 4 (red). The activity maps from IOI are overlaid with the fMRI response in the right part of Fig. 3 for this patient. Most of the areas that were activated within the fMRI are also visible using IOI, especially during the naming task with overt speech. The task with silent speech reveals mainly an area close to marker 7, where the speech arrest was induced by DES. The results were assessed as a moderate agreement between IOI and DES for silent speech and as nonagreement for overt speech. Comparing IOI and fMRI, a high agreement is visible for overt speech and a moderate agreement for silent speech (Table 3).

\section{IOI During the DES Mapping Procedure}

In 2 of 8 patients, we observed the intraoperative DES mapping procedure with IOI. We were able to visualize the spatial extent of metabolic changes after each DES and therefore the area that is affected through the stim- 
(A)

(B)

(C)

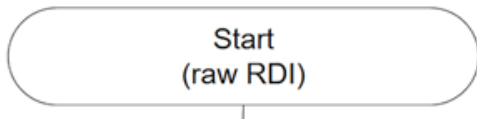

\begin{tabular}{|c|}
\hline $\begin{array}{c}\text { binarization / thresholding } \\
\text { (threshold } \mathrm{t}=0.02 \text { ) }\end{array}$ \\
\hline
\end{tabular}

symmetric gaussian lowpass filtering

$(5 \times 5$ Kernel, $\sigma=0.6)$

cleaning, closing, median filtering and segmentation of areas

(D)

(E)

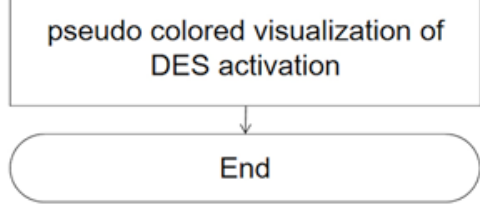

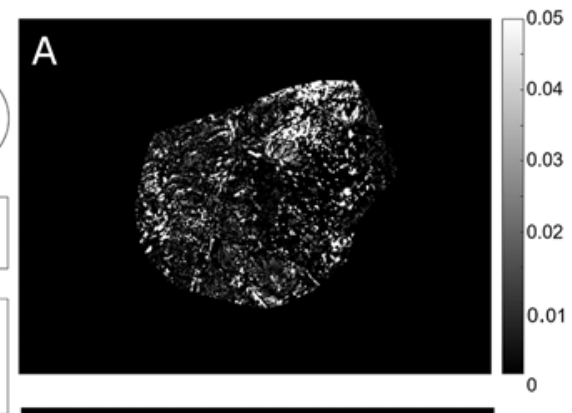
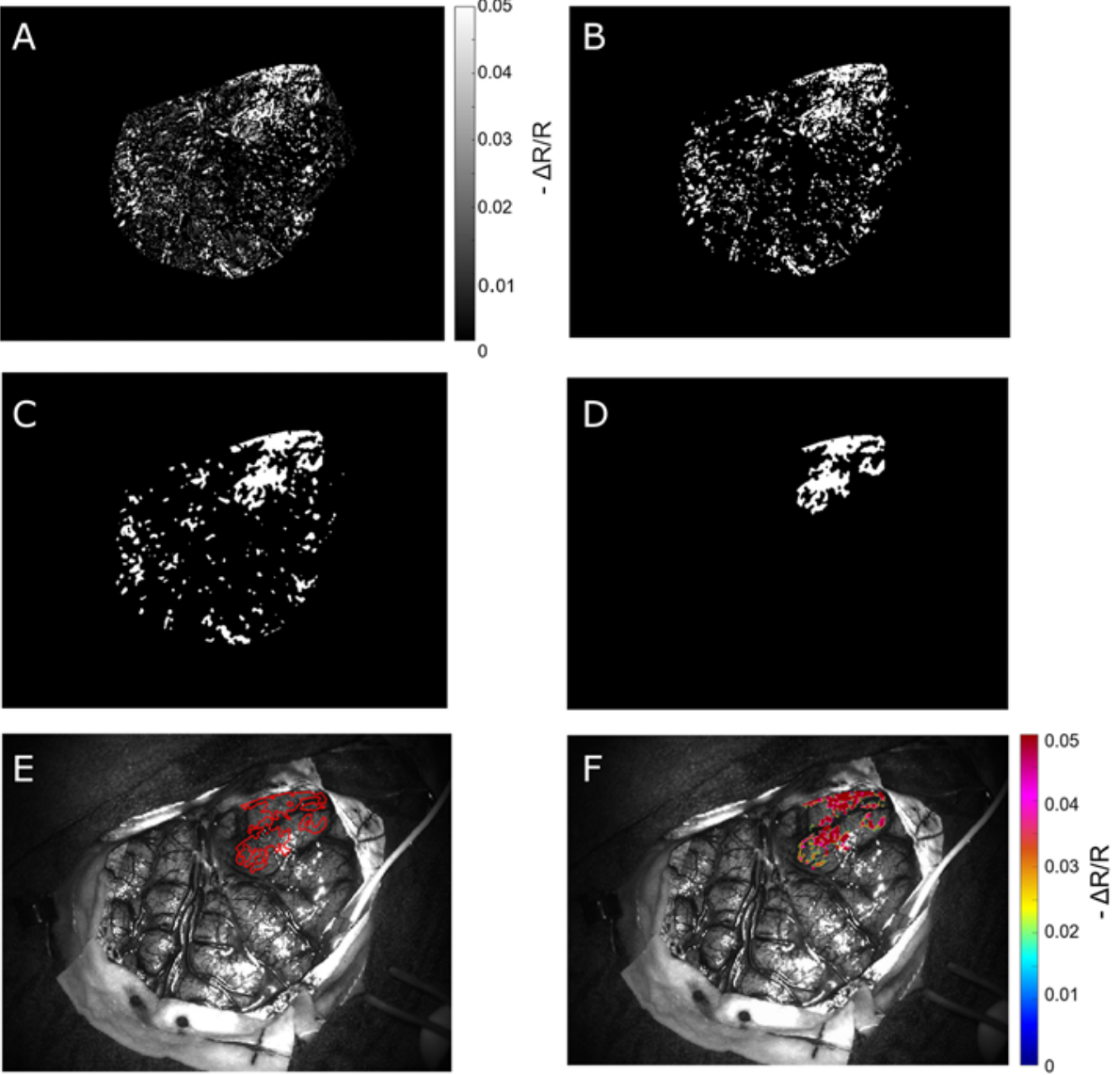

FIG. 1. Image processing chain for calculation of DES activation extent and pseudocolored signal amplitude based on the raw RDI between the rest and poststimulus phases. For the segmentation of the activated area, the point at the center of the bipolar electrodes $\left(\mathrm{p}_{\mathrm{COE}}\right)$ during electrical stimulation was used and only areas within a distance of 80 pixels (center of mass [ $\mathrm{p}_{\mathrm{CoM}}$ ]) were taken into account for the calculation of the extent of activated areas. $-\Delta R / R=$ normalized relative reflectance change: $\Delta R$ is the (mean) change of reflectivity during the stimulation period (with respect to the baseline) and $R$ is the (mean) baseline reflectivity.

ulation. A difference imaging technique in combination with thresholding and image processing techniques (see Methods section) was used. The results for the patients in cases 9 and 10 are shown in Fig. 4.

The extent of the stimulation-affected area varies in case 9 from $A_{\min }=56 \mathrm{~mm}^{2}$ to $A_{\max }=155 \mathrm{~mm}^{2}\left(\mathrm{~A}_{\text {mean }}=106\right.$ $\pm 30 \mathrm{~mm}^{2}[\mathrm{n}=8]$, area of trepanation $\left[\mathrm{A}_{\text {trep }}\right]=2626 \mathrm{~mm}^{2}$, amplitude of intraoperative stimulation current $\left[\mathrm{I}_{\text {stim }}\right]=6$ $\mathrm{mA})$. The highest extent of activation was observed at the site marked with the number 8 , at which a seizure was induced due to the electrical stimulation. The lowest extent of activation was observed at the site marked with number 1. At stimulation site number 9, afterdischarges were recorded and a diffuse, widespread activity is visible in the IOI activity map.

In case 10 (cortically located glioma), differences in the size of the activation area were observed between tumor and nontumor stimulation sites. At tumor stimulation sites $(n=3)$, the activation area varied between $A_{\min }=3 \mathrm{~mm}^{2}$ and $\mathrm{A}_{\max }=26 \mathrm{~mm}^{2}$, whereas at nontumor sites $(\mathrm{n}=4)$ the activated area ranged from $A_{\min }=44 \mathrm{~mm}^{2}$ to $A_{\max }=130$ $\mathrm{mm}^{2}\left(\mathrm{~A}_{\text {trep }}=1962 \mathrm{~mm}^{2}, \mathrm{I}_{\text {stim }}=6 \mathrm{~mA}\right)$.

\section{Discussion}

According to the findings, a promising field of application for IOI in awake procedures is the identification of primary motor areas on the precentral gyrus. Using a simple RGB camera, which is already part of modern surgical microscopes, we identified in 2 cases cortical areas in correspondence to the electrophysiological measurements. Contrary to the discrete information acquired with the electrode strips, IOI activity maps are spatially highly resolved. Additionally, activity maps have the methodologically inherent advantage that they can be easily semitransparently visualized over the actual intraoperative scene. Therefore, brain shift and the resulting loss of registration accuracy of the neuronavigation system are irrelevant for IOI, contrary to the preoperatively acquired fMRI for which the registration accuracy is of vital importance.

The comparison of fMRI and IOI for localization of eloquent language areas reveals in most cases significant differences between the modalities. There are several possible reasons that might contribute to those results. IOI, as well as fMRI, measures metabolic changes induced after neuronal activity through neurovascular coupling pro- 


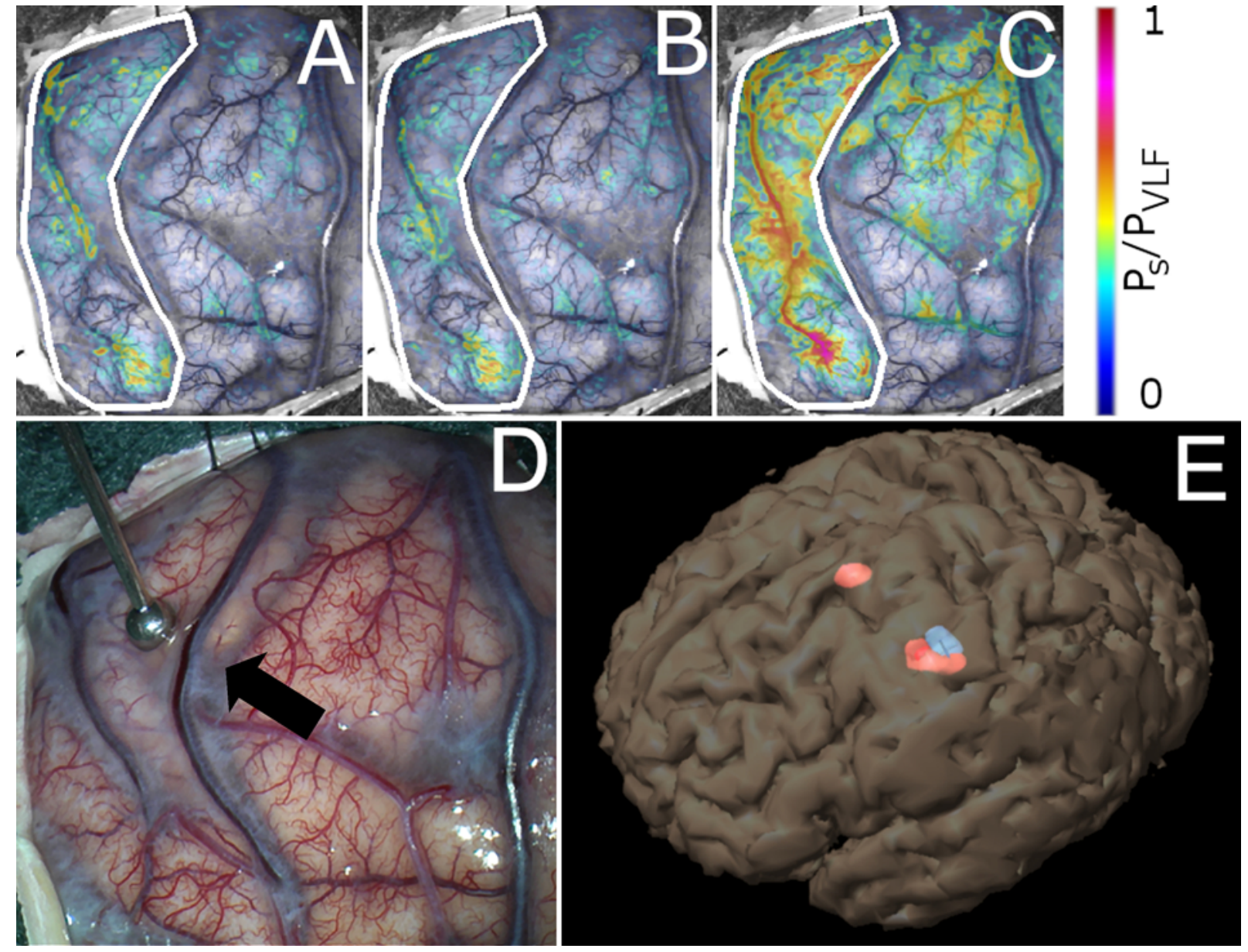

FIG. 2. Results of the finger-tapping task from the patient in case 1. A-C: IOI activity map calculated from blue (A), green (B), and red $(C)$ channel data of the charge-coupled device (CCD) color camera. The white line designates the contour of the precentral gyrus. The additional activation to the right of the motor area within the red channel map $(C)$ is located on the postcentral gyrus and may represent the sensory feedback component during motor task execution. D: Identification of precentral gyrus by monopolar cortex stimulation. Train-of-five pulses, $I_{\text {stim }}=5-20 \mathrm{~mA}$, interstimulation interval $(\mathrm{ISI})=4 \mathrm{msec}(250 \mathrm{~Hz}), \mathrm{T}_{\text {pulse }}=500 \mu$ sec. Black arrow indicates the site for the transsulcal approach to the metastasis. E: Screenshot of the neuronavigation map visualizing the tumor volume (blue) and the fMRI activation (red). $P_{s} / P_{V L F}=$ power spectral density/sum of the power spectral density within the very low-frequency band (stimulation frequency of $1 / 60 \mathrm{~Hz}$ for $\mathrm{P}_{\mathrm{s}} ; 2 \mathrm{D}$ map in both cases).

cesses. However, the physiological origin of the IOI signal is dependent on the observation wavelength. Pouratian et al. ${ }^{21}$ demonstrated that the fMRI blood oxygen level-dependent response shares a common etiology with the 610$\mathrm{nm}$ IOI signal. Nonetheless, the group observed differences in the colocalization of functional areas for IOI at 610 $\mathrm{nm}$ and fMRI as well as for fMRI and DES maps (tongue motor task). According to our experience from past studies, blood volume maps $(568 \mathrm{~nm}$ ) tend to be more compact and more strongly localized in the parenchyma than the oxygenation maps $(610 \mathrm{~nm})$, which emphasize larger venous structures and are more widespread. Therefore, we chose $568 \mathrm{~nm}$ as the observation wavelength for IOI in this investigation, but the results also reveal differences in the localization of activated areas for most of the cases. Despite the different physiological origin of the signal, the speech stimulation paradigm used might be a contributing factor to this difference in localization. During the fMRI measurements, silent speech was used to evoke activity in speech processing areas because the technique is susceptible to movement artifacts during the scanning process. In the operating room we performed silent speech in only 1 patient; otherwise (additionally in that patient), we used overt speech.

Using fMRI, several groups have already proven that different stimulation paradigms lead to different activated cortical sites. ${ }^{3,19}$ Therefore, we cannot neglect this influence, but the slight differences in the stimulation paradigm were accepted in this study because it is much easier for overt speech tasks to control whether the patient is cooperating (especially under the influence of anesthesia) and actually really performing the required task. For silent speech the missing feedback might lead to implausible or even no results, without the chance to reconstruct whether this was caused by a methodological problem, the underlying pathology/physiology, or just the lack of patient com- 

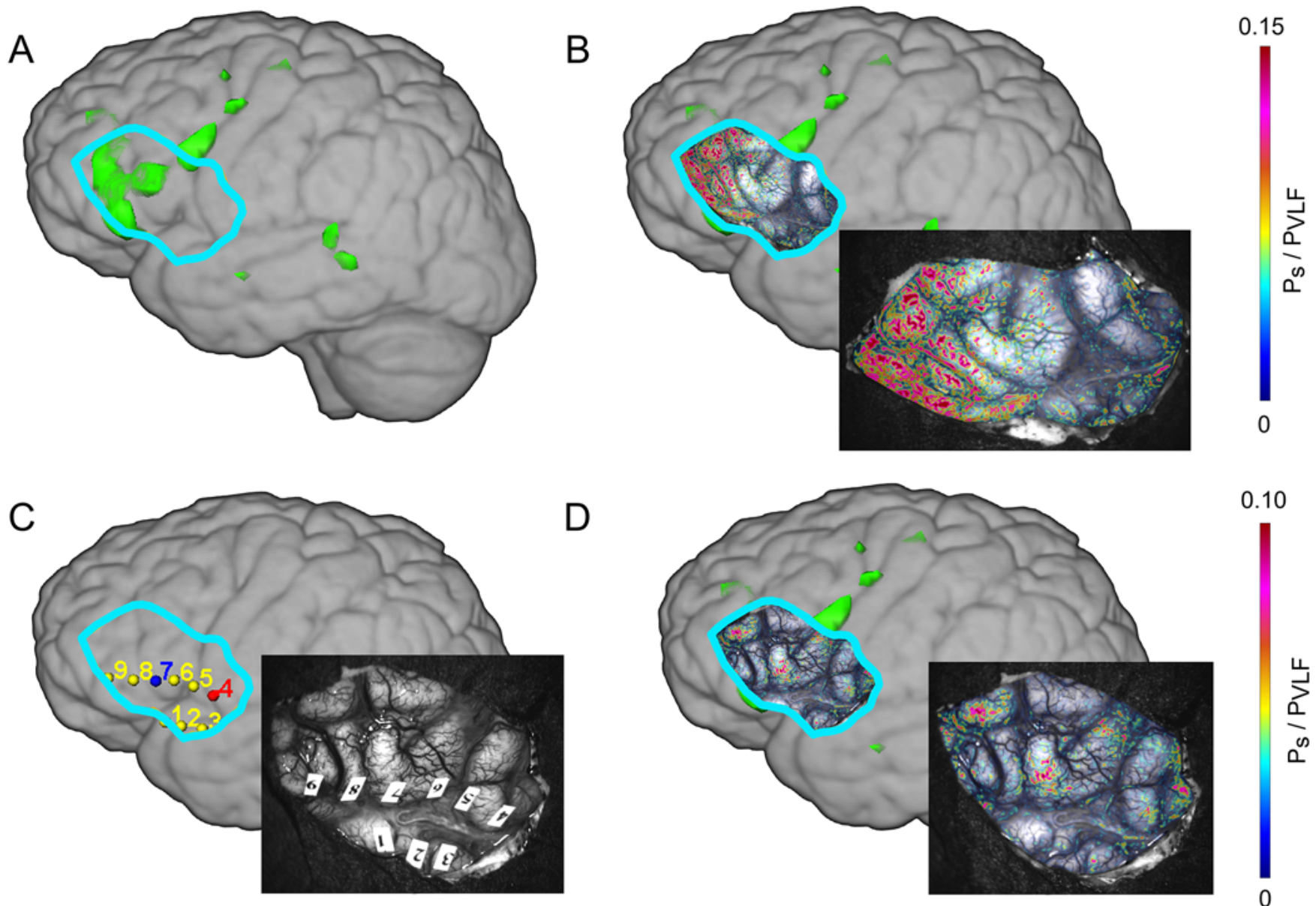

FIG. 3. Imaging results of the patient in case 3. A: fMRI activation. B: fMRI overlaid with IOI results for task with overt speech. C: DES markers (7 denotes speech arrest, 4 denotes seizure, precentral gyrus). D: fMRI overlaid with IOI results for task with silent speech.

pliance. The results in case 3 especially also reveal that despite the different stimulation paradigms a good agreement between fMRI (silent speech) and IOI (overt speech) is possible.

With respect to the results of the DES mapping, the activations of IOI as well as the activations of fMRI in this study were mostly larger than the area of the speech arrest sites, which is in line with the results of other studies published, e.g., by Cannestra et al. ${ }^{5}$ The findings lead to the conclusion that there is currently, according to our opinion and experience, little surgical benefit in applying IOI during surgery with speech tasks. Due to the complex nature of neuronal language processing, the interpretation of the data is challenging and the results are of little value for intraoperative decision-making.

Nevertheless, IOI can be valuable as a feedback and supplementary tool during language mapping. We used the method successfully to provide visual feedback to the surgeon about each spatial stimulation extent. The technique can be easily applied with little additional hardware and with minimal preoperative preparations. The only change in the workflow for the language mapping procedure with DES was the timing of the positioning of the numbered stimulation site markers. To ensure that they do not cover important regions of the surface, they were put on the cortex after the stimulation of the current site and the recording of the optical changes was finished.

As described in prior publications,${ }^{17} \mathrm{IOI}$ in connection with DES might also be able to assess if neurovascular coupling is intact or altered by pathological processes. The results from this investigation give more evidence to this hypothesis. The extent of the area activated through DES is significantly smaller on sites that were later identified as tumor areas (see results in case 8). This might be caused by neurovascular uncoupling and disruption of vascular regulation by the tumor. A closer look at the stimulations performed on what was most likely intact brain tissue of the patient in case 1 reveals the diversity of the area affected through the DES. Although the mean area for all stimulations is in line with the expectation of approximately 1 $\mathrm{cm}^{2}$, the standard deviation reveals a high interstimulation variability, ranging from approximately $50 \%$ up to approximately $150 \%$ of the estimated area. Furthermore, the optical changes and blood volume changes are not arranged symmetrically around the electrode tips but are altered by micro- and macroanatomical factors, e.g., vessels and the gyral structure. Therefore, a visual feedback for each stimulation is beneficial for the mapping optimization. 
A
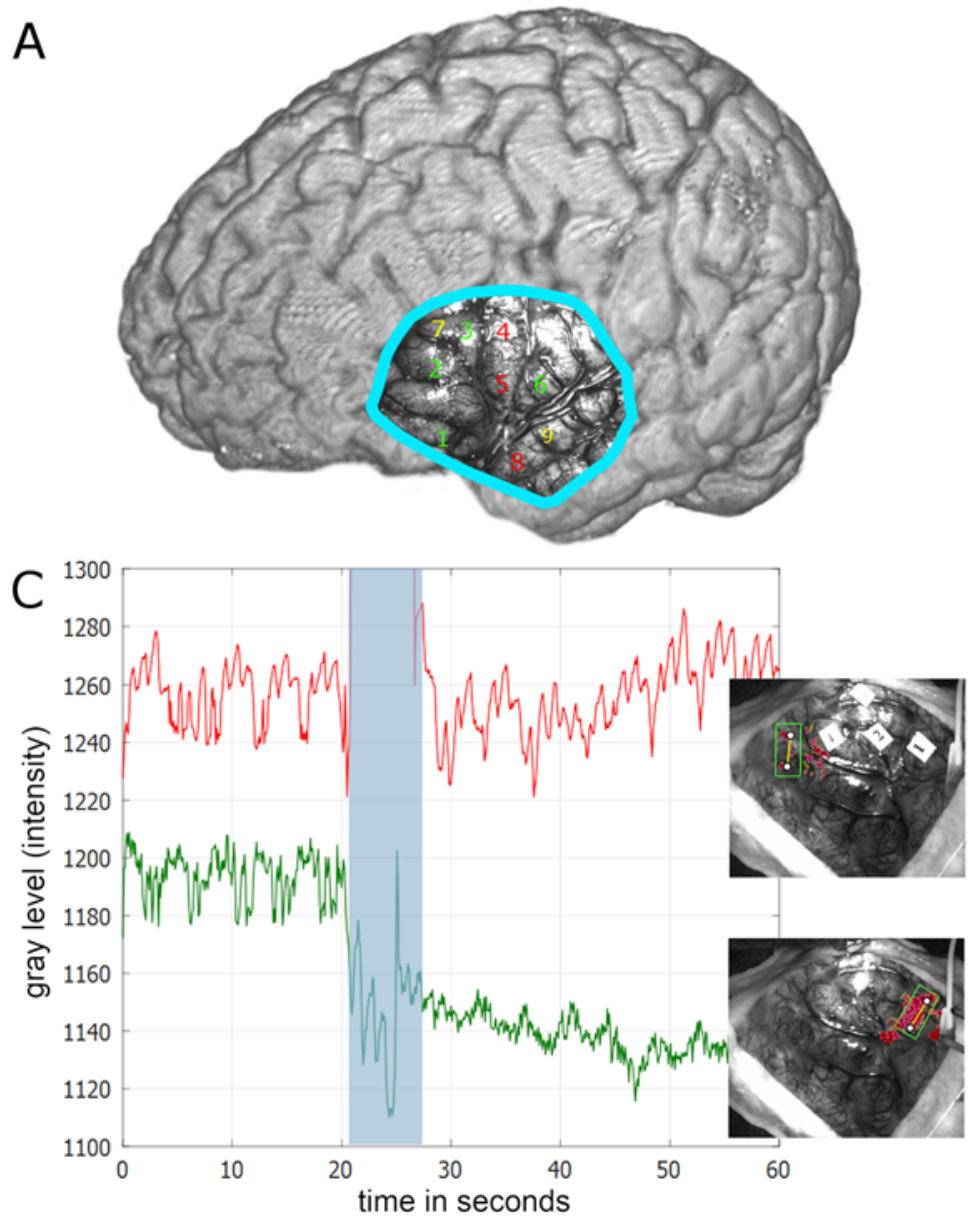

$\mathrm{B}$
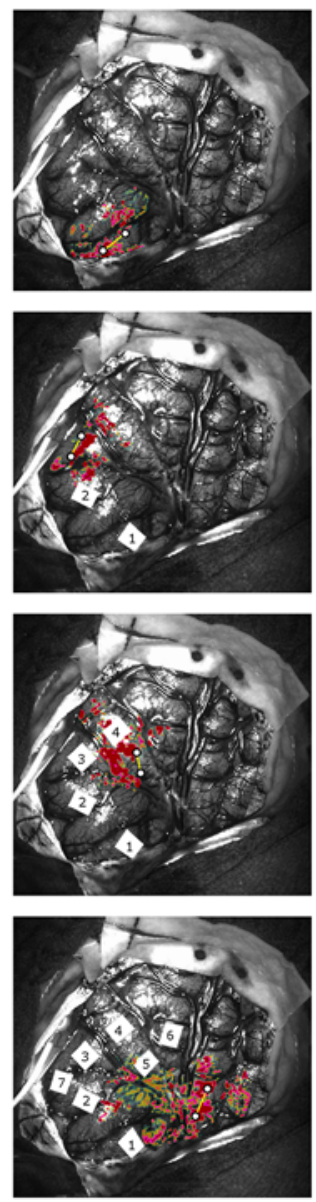
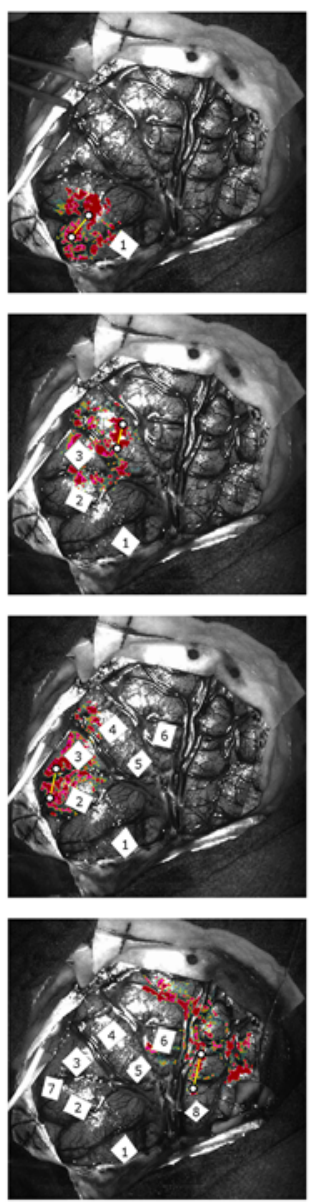

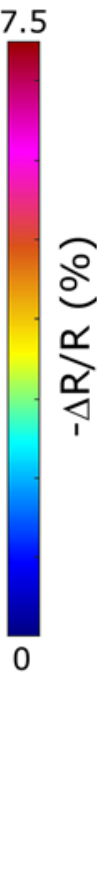

FIG. 4. Results of DES mapping procedure for the patients in cases 9 and 10. A: Brain surface reconstruction of case 9 based on preoperative MRI data overlaid with intraoperative image $(568 \mathrm{~nm})$ and stimulation position markers; speech arrest at 4 and 5 , and seizure at 8 (red numbers); reduced speaking ability at 7 and afterdischarges at 9 (yellow numbers). B: Visualized spatial extent for each stimulation in case 9; numbered markers were put on the surface after each stimulation. C: Intensity raw time courses for a stimulation on tumor tissue (red line in graph) and nontumor tissue (green line in graph) of case 10. The time courses were averaged from the region of interest shown overlaid on the activity map of the stimulations on the right side. During the stimulation (grayed out) the electrodes covered the brain surface; therefore, there is no useful information available from this period. A significant drop of the intensity (increased CBV) with respect to the baseline is clearly visible on nontumor tissue, whereas on tumor tissue between baseline and the poststimulus phase no difference can be observed.

The results are promising but must be interpreted carefully because the underlying physiological mechanisms of DES are still poorly understood, subject to ongoing discussions $s^{4,7}$ and dependent on several technical factors (e.g., observation wavelength ${ }^{33}$ ). It is still unclear how close the observed metabolic changes and the electrical field propagation are located to each other. The relative difference imaging technique is furthermore susceptible for reflections and movement of the cortical surface. Therefore, the microscope with the attached camera should be positioned in a rather flat angle toward the cortical surface to avoid specular reflections. Because the patient is awake and speaking during the procedure, problems can also arise from the small movements that are present even though the head is fixed in the stereotactic frame. Therefore, we performed an elastic image registration of each time series to minimize the motion influences during the image data evaluation. The weaknesses of the current study are the small and diverse patient cohort as well as the fact that we only performed a qualitative comparison between the different modalities. We found this to be sufficient for an initial evaluation of IOI during language and motor testing. According to our findings, the results from the 9-minute speech task are too unspecific for a quantitative comparison that in turn is also highly dependent on multiple influencing factors like fMRI significance thresholds and activation depth, IOI visualization thresholds, and DES parameters.

\section{Conclusions}

According to the findings, the activity maps of the 9-minute speech tasks are too unspecific to provide reliable information about the essential speech areas that need to be preserved during the surgical procedure. Therefore, brain mapping with DES will remain the method of 
choice. A more useful application for IOI is the identification of primary motor areas. We were able to map those areas successfully in 2 patients in correspondence to fMRI and electrophysiological measurements by using a simple color camera.

A promising new application of IOI is its combined use with DES during the standard language mapping procedure. Here, the technique is able to provide visual feedback about the extent of the stimulated area and about the metabolic changes induced through the stimulation, which may correspond with the functional state of neurovascular coupling. For each stimulation, the visualization can be computed within 90 seconds (including the image acquisition time). This helps the surgeon to estimate the individual extent of each stimulation, optimize the mapping procedure, and identify nonactivated areas as functionally impaired tumor tissue. Nevertheless, to prove and verify the presented results, additional extensive investigationsespecially regarding the multiple influencing parameters of the hardware and evaluation software-are mandatory. Further work should focus on the acquisition of new patient data and the in-depth analysis of the most promising IOI applications in awake surgery, applications comprising visualization of motor cortex activation and the stimulation extent during brain mapping.

\section{Acknowledgments}

We thank Andreas Schöppe, Anita Menschner, and Enrico Noback for their excellent technical assistance and support.

\section{References}

1. Bangiyev L, Rossi Espagnet MC, Young R, Shepherd T, Knopp E, Friedman K, et al: Adult brain tumor imaging: state of the art. Semin Roentgenol 49:39-52, 2014

2. Bauer AQ, Kraft AW, Wright PW, Snyder AZ, Lee JM, Culver JP: Optical imaging of disrupted functional connectivity following ischemic stroke in mice. Neuroimage 99:388-401, 2014

3. Binder JR, Swanson SJ, Hammeke TA, Sabsevitz DS: A comparison of five fMRI protocols for mapping speech comprehension systems. Epilepsia 49:1980-1997, 2008

4. Borchers S, Himmelbach M, Logothetis N, Karnath HO Direct electrical stimulation of human cortex - the gold standard for mapping brain functions? Nat Rev Neurosci 13:63-70, 2011

5. Cannestra AF, Bookheimer SY, Pouratian N, O'Farrell A, Sicotte N, Martin NA, et al: Temporal and topographical characterization of language cortices using intraoperative optical intrinsic signals. Neuroimage 12:41-54, 2000

6. Cannestra AF, Pouratian N, Forage J, Bookheimer SY, Martin NA, Toga AW: Functional magnetic resonance imaging and optical imaging for dominant-hemisphere perisylvian arteriovenous malformations. Neurosurgery 55:804-814, 2004

7. Desmurget M, Song Z, Mottolese C, Sirigu A: Re-establishing the merits of electrical brain stimulation. Trends Cogn Sci 17:442-449, 2013

8. Dimou S, Battisti RA, Hermens DF, Lagopoulos J: A systematic review of functional magnetic resonance imaging and diffusion tensor imaging modalities used in presurgical planning of brain tumour resection. Neurosurg Rev 36:205-214, 2013

9. Fink JR, Muzi M, Peck M, Krohn KA: Multimodality brain tumor imaging: MR imaging, PET, and PET/MR imaging. J Nucl Med 56:1554-1561, 2015

10. Haglund MM, Berger MS, Hochman DW: Enhanced optical imaging of human gliomas and tumor margins. Neurosurgery 38:308-317, 1996

11. Haglund MM, Ojemann GA, Hochman DW: Optical imaging of epileptiform and functional activity in human cerebral cortex. Nature 358:668-671, 1992

12. Lu HD, Roe AW: Functional organization of color domains in V1 and V2 of macaque monkey revealed by optical imaging. Cereb Cortex 18:516-533, 2008

13. Mabray MC, Barajas RF Jr, Cha S: Modern brain tumor imaging. Brain Tumor Res Treat 3:8-23, 2015

14. Mongen MA, Willems PWA: Current accuracy of surface matching compared to adhesive markers in patient-to-image registration. Acta Neurochir (Wien) 161:865-870, 2019

15. Morone KA, Neimat JS, Roe AW, Friedman RM: Review of functional and clinical relevance of intrinsic signal optical imaging in human brain mapping. Neurophotonics 4:031220, 2017

16. Nariai T, Sato K, Hirakawa K, Ohta Y, Tanaka Y, Ishiwata $\mathrm{K}$, et al: Imaging of somatotopic representation of sensory cortex with intrinsic optical signals as guides for brain tumor surgery. J Neurosurg 103:414-423, 2005

17. Oelschlägel M, Meyer T, Schackert G, Kirsch M, Sobottka $\mathrm{SB}$, Morgenstern U: Intraoperative optical imaging of metabolic changes after direct cortical stimulation-a clinical tool for guidance during tumor resection? Biomed Tech (Berl) 63:587-594, 2018

18. Oelschlägel M, Meyer T, Wahl H, Sobottka SB, Kirsch M, Schackert G, et al: Evaluation of intraoperative optical imaging analysis methods by phantom and patient measurements. Biomed Tech (Berl) 58:257-267, 2013

19. Petrovich Brennan NM, Whalen S, de Morales Branco D, O'Shea JP, Norton IH, Golby AJ: Object naming is a more sensitive measure of speech localization than number counting: converging evidence from direct cortical stimulation and fMRI. Neuroimage 37 (Suppl 1):S100-S108, 2007

20. Pouratian N, Bookheimer SY, O'Farrell AM, Sicotte NL, Cannestra AF, Becker D, et al: Optical imaging of bilingual cortical representations. Case report. J Neurosurg 93:676681,2000

21. Pouratian N, Sicotte N, Rex D, Martin NA, Becker D, Cannestra AF, et al: Spatial/temporal correlation of BOLD and optical intrinsic signals in humans. Magn Reson Med 47:766-776, 2002

22. Prakash N, Uhlemann F, Sheth SA, Bookheimer S, Martin N, Toga AW: Current trends in intraoperative optical imaging for functional brain mapping and delineation of lesions of language cortex. Neuroimage 47 (Suppl 2):T116-T126, 2009

23. Sato K, Nariai T, Momose-Sato Y, Kamino K: Intraoperative intrinsic optical imaging of human somatosensory cortex during neurosurgical operations. Neurophotonics 4:031205, 2017

24. Sato K, Nariai T, Sasaki S, Yazawa I, Mochida H, Miyakawa $\mathrm{N}$, et al: Intraoperative intrinsic optical imaging of neuronal activity from subdivisions of the human primary somatosensory cortex. Cereb Cortex 12:269-280, 2002

25. Sato K, Nariai T, Tanaka Y, Maehara T, Miyakawa N, Sasaki $\mathrm{S}$, et al: Functional representation of the finger and face in the human somatosensory cortex: intraoperative intrinsic optical imaging. Neuroimage 25:1292-1301, 2005

26. Schwartz TH, Chen LM, Friedman RM, Spencer DD, Roe AW: Intraoperative optical imaging of human face cortical topography: a case study. Neuroreport 15:1527-1531, 2004

27. Silva MA, See AP, Essayed WI, Golby AJ, Tie Y: Challenges and techniques for presurgical brain mapping with functional MRI. Neuroimage Clin 17:794-803, 2017

28. Sobottka SB, Meyer T, Kirsch M, Koch E, Steinmeier R, 
Morgenstern U, et al: Evaluation of the clinical practicability of intraoperative optical imaging comparing three different camera setups. Biomed Tech (Berl) 58:237-248, 2013

29. Sobottka SB, Meyer T, Kirsch M, Koch E, Steinmeier R, Morgenstern U, et al: Intraoperative optical imaging of intrinsic signals: a reliable method for visualizing stimulated functional brain areas during surgery. J Neurosurg 119:853863,2013

30. Sobottka SB, Meyer T, Kirsch M, Reiss G, Koch E, Morgenstern U, et al: Assessment of visual function during brain surgery near the visual cortex by intraoperative optical imaging. Biomed Tech (Berl) 58:249-256, 2013

31. Song Y, Riera JJ, Bhatia S, Ragheb J, Garcia C, Weil AG, et al: Intraoperative optical mapping of epileptogenic cortices during non-ictal periods in pediatric patients. Neuroimage Clin 11:423-434, 2016

32. Stieglitz LH, Fichtner J, Andres R, Schucht P, Krähenbühl AK, Raabe A, et al: The silent loss of neuronavigation accuracy: a systematic retrospective analysis of factors influencing the mismatch of frameless stereotactic systems in cranial neurosurgery. Neurosurgery 72:796-807, 2013

33. Suh M, Bahar S, Mehta AD, Schwartz TH: Blood volume and hemoglobin oxygenation response following electrical stimulation of human cortex. Neuroimage 31:66-75, 2006

34. Wang G, Tanifuji M, Tanaka K: Functional architecture in monkey inferotemporal cortex revealed by in vivo optical imaging. Neurosci Res 32:33-46, 1998

35. Wang Z, Chen LM, Négyessy L, Friedman RM, Mishra A, Gore JC, et al: The relationship of anatomical and functional connectivity to resting-state connectivity in primate somatosensory cortex. Neuron 78:1116-1126, 2013

36. White BR, Bauer AQ, Snyder AZ, Schlaggar BL, Lee JM, Culver JP: Imaging of functional connectivity in the mouse brain. PLoS One 6:e16322, 2011

\section{Disclosures}

Mr. Oelschlägel received PhD funding from Carl Zeiss Meditec AG, Oberkochen, Germany. Additionally, material support (camera systems, optical components) was provided by Carl Zeiss Meditec AG.

\section{Author Contributions}

Conception and design: Oelschlägel, Sobottka. Acquisition of data: Oelschlägel, Meyer, Wahl, Gerber, Reiß, Kirsch, Sobottka. Analysis and interpretation of data: Oelschlägel, Meyer, Wahl, Gerber, Sobottka. Drafting the article: Oelschlägel, Sobottka. Critically revising the article: Oelschlägel, Morgenstern, Koch, Steiner, Schackert, Sobottka. Reviewed submitted version of manuscript: all authors. Approved the final version of the manuscript on behalf of all authors: Oelschlägel. Statistical analysis: Oelschlägel, Wahl, Gerber. Administrative/technical/material support: Morgenstern, Koch, Steiner, Schackert. Study supervision: Schackert, Sobottka.

\section{Supplemental Information Videos}

Video Abstract. https://vimeo.com/384072722.

\section{Previous Presentations}

Portions of this work were presented in abstract and oral presentation form at the German conference Jahrestagung Sektion Neuroonkologie der Deutschen Gesellschaft für Neurochirurgie (DGNC), Innsbruck, Austria, November 9-10, 2018.

\section{Correspondence}

Martin Oelschlägel: Technische Universität Dresden, Saxony, Germany.martin.oelschlaegel@tu-dresden.de. 